\title{
Highly efficient detoxification of dinitrotoluene through overexpressing bacterial nitroreductase in switchgrass
}

\author{
Kunlong $\mathrm{Su}^{1}$, Zhenying $\mathrm{Wu}^{1}$, Yuchen $\mathrm{Liu}^{1}$, Shanshan Jiang ${ }^{1}$, Yan Wang ${ }^{1}$, and Chuanxiang \\ $\mathrm{Fu}^{1}$ \\ ${ }^{1}$ Qingdao Institute of BioEnergy and Bioprocess Technology Chinese Academy of Sciences
}

February 14, 2021

\begin{abstract}
Dinitrotoluene (DNT) has been extensively used in manufacturing munitions, polyurethane foams, and other important chemical products. However, it is highly toxic and mutagenic to most organisms. Here, we synthesized a codon optimized bacterial nitroreductase gene, NfsI, for plant expression. The kinetic analysis indicates that the recombinant NfsI can detoxify both 2,4DNT and its sulfonate (DNTS), while it has a 97.6-fold higher catalytic efficiency for 2,4-DNT than DNTS. Furthermore, we overexpressed NfsI in switchgrass (Panicum virgatum L.), which is a multiple purpose crop used for fodder and biofuel production as well as phytoremediation. The 2,4-DNT treatment inhibited root elongation of wild type switchgrass plants and promoted reactive oxygen species (ROS) accumulation in roots. In contrast, overexpression of NfsI in switchgrass significantly alleviated 2,4-DNT-induced root growth inhibition and ROS overproduction. Thus, the NfsI overexpressing transgenic switchgrass plant removed 94.1\% 2,4-DNT after 6 days, whose efficiency was 1.7-fold higher than control plants. Moreover, the comparative transcriptome analysis suggests that $22.9 \%$ of differentially expressed genes induced by 2,4-DNT may participate in NfsImediated 2,4-DNT detoxification in switchgrass. Our work sheds light on the function of NfsI during DNT phytoremediation for the first time, revealing the application potential of switchgrass plants engineered with NfsI.
\end{abstract}

\section{Introduction}

Dinitrotoluene (DNT) is a nitroaromatic compound extensively used in manufacturing munitions, polyurethane foams, automobile airbags, and other important chemical products (Rickert et al., 1984). Due to a history of long-term manufacturing, civilian and military use, and waste steam leaks, DNT has entered the soil and water, which threatens the health of residents and the environment around DNT manufacturing and processing facilities. DNT exists as six isomers, with 2,4- and 2,6-DNT being the major forms that are moderately to highly toxic to humans, animals, and plants (Gong et al., 2003). The mixture of 2,4- and 2,6-DNT has been classified as a Class B2 carcinogen to humans and animals by the United States Environmental Protection Agency (EPA). Among them, 2,4-DNT is predominantly employed as an intermediate for production of polyurethane (Lent et al., 2012). It is also a byproduct from the synthesis of 2,4,6-trinitrotoluene (TNT), which is one of the most widely used explosives used for civilian and military purposes around the world. In addition, 2,4-dinitrotoluene-3-sulfonate (DNTS) produced from TNT refining is a major toxic component in TNT red water and its contaminated soils (Tsai, 1991). Both 2,4-DNT and its sulfonate have long-term environmental persistence and high-energetic toxicity and mutagenicity to most organisms (Gilbert, 1977). Thus, they have been considered as the priority pollutants that must be removed from soil and groundwater contaminated by manufacturing and processing of polyurethane and TNT.

Biological remediation including phytoremediation and microbial remediation is a safe, economic, and highly efficient strategy for degradation of nitroaromatic compounds. Previous studies have suggested that the detoxification pathway of DNT and other nitroaromatic explosives in plants might go through three phases: functionalization (hydrolysis, oxidation, and reduction), transformation (conjugation by glycosyl transferases 
or glutathione transferases), and compartmentalization (transportation to cell wall or vacuole) (Rai et al., 2020; Rao et al., 2009). However, the detailed molecular mechanism of detoxification of these nitroaromatic compounds remains elusive in plants. Previous studies have suggested that the high toxicity of nitroaromatic compounds to plants results from the predominant accumulation of excessive reactive oxygen species (ROS) in plant tissues, which damages normal physiological activities in plant cells (Brentner et al., 2010; Johnston et al., 2015). Deficiency of Arabidopsis MONODEHYDROASCORBATE REDUCTASE 6 (MDHAR6) can reduce the production of ROS in the mitochondria and confer Arabidopsishigh tolerance to TNT (Johnston et al., 2015). In addition, overexpression of UDP-glycosyltransferases (UGT743B4 and UGT73C1 ) and glutathione transferases (GSTU24 and GSTU25 ) in Arabidopsis leads to accumulation of the conjugation products of TNT and reduces its cytotoxicity (Gandia-Herrero et al., 2008; Gunning et al., 2014). Although some efforts have been made to alleviate the pollution of TNT by phytoremediation over recent decades, only one bioengineering work has been made for DNT detoxification by expressing a cyanobacterial flavodoxin in tobacco plants (Tognetti et al., 2007).

In contrast, DNT detoxification microbes and their degradation mechanism have been investigated extensively. Many microbes includingEnterobacter cloacae ,Saccharomyces sp, Cadida sp, and white-rot fungus participate in DNT degradation, which provide enriched sources for identification and characterization of genes involved in detoxification of these nitroaromatic compounds (Koder and Miller, 1998; Ziganshin et al., 2007; Kist et al., 2020). Previous studies have suggested that a dioxygenase is responsible for the first step of the DNT degradation pathway. However, this dioxygenase is encoded by four genes ( $\operatorname{dnt} A a, A b, A c$, $A d$ ) in Burkholderia cepacian R34, which makes it difficult to be applied for plant bioengineering (Johnson et al., 2002). Microbial type I nitroreductase (NR) is an oxygen-insensitive flavoprotein that catalyzes the $\mathrm{NAD}(\mathrm{P}) \mathrm{H}$-dependent reduction of nitro groups to hydroxylamino and/or amino groups on nitroaromatic compounds. These type I NRs consist of two main groups, NfsA (group A) and NfsB (group B) (Roldán et al., 2008). Moreover, members of NfsB can reduce both 2,4- and 2,6-DNT (Williams et al., 2019), while members of NfsA only convert 2,4-DNT (Rich et al., 2018). NfsI is a member of NfsB NR identified fromEnterobacter cloacae (Zajc, 1999). The recombinant NfsI protein can convert both TNT and DNT in vitro (Bryant et al., 1991). Overexpression of $N f s I$ successfully improves the degradation efficiency of TNT in transgenic poplar, tobacco, and wheatgrass (Brentner et al., 2010; Zhang et al., 2017b; Zhang et al., 2019). These findings shed light on phytoremediation through transgenic plants engineered with bacterial nitroaromatic compound degradation genes. However, it is still unknown if the plants engineered with $N f_{S} I$ could detoxify DNT and its sulfonates.

Switchgrass (Panicum virgatum L.) is a perennial C4 tall grass that has been used as lignocellulosic feedstock for forage and biofuel production. Switchgrass can increase soil organic carbon and facilitate a larger microbe population in margin lands. Moreover, switchgrass is well adapted to various soil types with excellent drought, cold, saline, and heavy metal tolerance (Song et al., 2018). Therefore, switchgrass has great potential for phytoremediation because of its extensive root system, vigorous growth, high ability to stress tolerance, and low-input requirements (Rai et al., 2020). A recent study has shown that ectopic expression of bacterial flavodoxin-cytochrome P450 XplA coupled with flavodoxin reducatase $X p l B$ in switchgrass can dramatically improve the removal efficiency of RDX in transgenic plants (Zhang et al., 2017a). Unfortunately, overexpression of the bacterial $N f_{s} I$ in switchgrass cannot degrade TNT due to low transcription of NfsI(Zhang et al., 2017a).

Here, we synthesized a codon optimized bacterial $N f_{s} I$ for overexpression in switchgrass and studied its function in DNT and DNTS detoxification for the first time. The transgenic switchgrass with NfsI overexpression significantly alleviated the 2,4-DNT-induced root growth inhibition and reduced ROS content. The removal efficiency for DNT achieved $94.1 \%$ in $N f s I$ overexpressing transgenic switchgrass plants, which was 1.7-fold higher than that of control plants. In contrast, overexpression of $N f_{s I}$ in switchgrass barely improved the removal capacity for DNTS, supporting the in vitro enzyme kinetics analysis that suggests that the recombinant NfsI has approximate 100-fold higher catalytic efficiency for 2,4-DNT than DNTS. Furthermore, overexpression of $N f s I$ in switchgrass partially alleviated the impact of 2,4-DNT on expression profiling of genes involved in plant detoxification. Our work suggests that engineering $N f_{s} I$ in plants may have great 
application potential for DNT phytoremediation in the future.

\section{Materials and Methods}

\section{Plant materials and growth conditions}

A lowland-type switchgrass cultivar, Alamo, was employed for detoxification of DNT and DNTS. According to the criteria described by Hardin (Hardin et al. 2013), the development of our switchgrass plants at vegetative phase was divided into three vegetative stages (V1, V2, and V3) and five elongation stages (E1, E2, E3, E4, and E5) before they entered the reproductive phase. Plants were grown in a greenhouse with a duration period of 16-hours of light $\left(390 \mu \mathrm{E} \cdot \mathrm{m}^{-2} \cdot \mathrm{S}^{-1}\right)$.

\section{Codon optimization of $N f s I$}

The coding sequence of $N f s I$ characterized in Enterobater cloacae was downloaded from GenBank (accession No. M6308.1) (Bryant and DeLuca, 1991). The codon optimization of NfsI was based on the code preference of switchgrass and was subjected to chemical synthesis.

\section{Enzyme kinetics analysis of recombinantNfsI}

The codon optimized $N f s I$ was cloned into pET32a vector after digested by Eco RI. The recombinant plasmid pET32a-NfsI was transformed into Rosetta E. coli for production of recombinant NfsI protein (Xiong et al., 2019). The purified recombinant protein was subjected to enzyme kinetics analysis of NfsI against 2,4DNT and DNTS, as described by Bryant (Bryant and DeLuca, 1991). Moreover, 2,4-DNT, DNTS, and their transformed products were identified and quantified by reversed phase high-performance liquid chromatography along with a photo diode array and electrospray ionization tandem mass spectrometry (LC-PDA/ESIMS/MS). The authentic 2,4-DNT and DNTS were used as external criteria to identify the 2,4-DNT and DNTS, and their content was quantitated based on the absorbance at $254 \mathrm{~nm}$ and $205 \mathrm{~nm}$, respectively. The 2,4-DNT and DNTS were ordered from Aladdin Industrial Corporation, Shanghai, China (CAS No. 121-14-2) and Synchem OHG, Germany (CAS No. 63348-71-0), respectively.

\section{Enzymatic activity assay of NfsI at different $\mathrm{pH}$ values and NADPH concentrations}

The effects of $\mathrm{pH}$ and NADPH on NfsI activity were determined by the methods described by Kim (Kim and Song, 2005). NfsI activities were examined after being incubated with reaction buffers at $\mathrm{pH}$ 4.0, 5.0, 6.0, 7.0, 8.0, 9.0. The reaction mixture contained $50 \mu \mathrm{g}$ purified recombinant NfsI protein, $200 \mu \mathrm{M}$ 2,4-DNT/DNTS, $200 \mu \mathrm{M} \mathrm{NADPH}$, and $50 \mathrm{mM}$ of sodium phosphate buffer in the final volume of $500 \mu \mathrm{L}$ with a pH range of 4.0-9.0. NfsI activities were also detected after being incubated with reaction buffers containing different NADPH concentrations $(50,100,200,400,800,1600 \mu \mathrm{M})$. The reaction mixture contained $50 \mu \mathrm{g}$ purified recombinant NfsI protein, $200 \mu \mathrm{M}$ 2,4-DNT/DNTS, $50 \mathrm{mM}$ sodium phosphate buffer (pH 6.0), and different $\mathrm{NADPH}$ concentrations in the final volume of $500 \mu \mathrm{L}$. After $30 \mathrm{~min}$ of incubation at $30{ }^{\circ} \mathrm{C}$, the enzymatic reaction was terminated by $50 \mu \mathrm{L}$ methanol, and the mixture was centrifuged at 17,000 g for $10 \mathrm{~min}$. The supernatant was collected and filtered through $0.22 \mu \mathrm{m}$ filter membrane for HPLC analysis.

\section{Generation of $N f s I$ overexpressing transgenic switchgrass plants}

The codon optimized $N f s I$ sequence was cloned into pENTR vector infused with cMyc tag and then subcloned into pANIC6B vector via attL-attR recombinant reaction (Mann et al., 2012). The $N f_{s} I$ overexpression vector pANIC6B_NfsI_cMyc was finally transformed into_fgrobacterium tumefaciens strain EHA105 using the freeze-thaw method (Wise et al., 2006). The single genotype callus line induced from switchgrass cultivar Alamo was employed for Agrobacterium -mediated transformation, following the procedure as described by $\mathrm{Wu}$ (Wu et al., 2016). Hygromycin was used as the selectable reagent to generate NfsI overexpressing transgenic switchgrass lines that were transplanted into soils and grown in the greenhouse. The control plants were generated with the pANIC6B empty vector.

Expression levels of $N f s I$ in transgenic switchgrass plants 
The independent positive transgenic lines were identified by genomic PCR with specific $h p h$ and $N f s I$ primers. The expected sizes of PCR products were 375 and 654 bp for $h p h$ and $N f s I$ (Table S1). Stems at the E2 stage were collected from each plant and ground in liquid nitrogen. Approximate $200 \mathrm{mg}$ stem samples were extracted for total RNA by TriZol extraction kits (TransGen Biotech, China) and subjected to reverse transcription with SuperMix (TransGen Biotech, China) after treatment with TURBO DNase I (Ambion, Austin, TX). The expression levels of $N f s I$ were analyzed by quantitative real-time PCR (qRT-PCR). The primers used for qRT-PCR are listed in Table S1. The cycle thresholds were determined using a ABI PRISM 7900 HT sequence detection system (Applied Biosystems, Foster City, CA), and the data were normalized using the level of switchgrass PvUbq2 transcripts (GenBank accession No. HM209468).

\section{Root length and ROS content}

The two representative transgenic switchgrass lines, control, and wild type plants were micropropagated by node culture described by Alexandrova (Alexandrova et al., 1996). The well rooted uniform regenerated plantlets were subcultured in new 1/2 MS solid medium after their roots were cut off. Thereafter, the wild type plantlets were treated with different concentrations of 2,4-DNT $\left(0,2,5,10,20,40 \mathrm{mg} \cdot \mathrm{L}^{-1}\right)$ and DNTS $\left(0,2,4,8,16 \mathrm{~g} \cdot \mathrm{L}^{-1}\right)$, respectively. The control and transgenic switchgrass plantlets were further treated with $20 \mathrm{mg} \cdot \mathrm{L}^{-1} 2,4$-DNT. The roots of switchgrass plantlets treated with 2,4-DNT and DNTS were photographed, and their length was measured after two weeks of growth. An enzyme-linked immunosorbent assay (ELISA) was employed for detecting ROS contents of switchgrass plantlets. Approximately $50 \mathrm{mg}$ of the above fresh root samples were ground in the sodium phosphate buffer (0.01 M, pH 7.4). After centrifugation at 4,500 g,

$4{ }^{\circ} \mathrm{C}$ for $20 \mathrm{~min}$, the supernatants were collected for measurement of ROS contents following the protocol of ELISA kit (Shanghai Enzyme-linked Biotech, China). The DAB stain following the procedure was carried out as described by Johnston (Johnston et al., 2015).

\section{2,4-DNT and DNTS uptake by control and transgenic switchgrass plants}

The control and transgenic switchgrass plants were propagated through tiller-splitting. The uniform switchgrass plants at E2 stage were selected and transplanted in $200 \mathrm{~mL} \mathrm{1/2} \mathrm{MS} \mathrm{liquid} \mathrm{medium} \mathrm{without} \mathrm{sugar}$ and supplied with $20 \mathrm{mg} \cdot \mathrm{L}^{-1} 2,4-\mathrm{DNT}$ and $1 \mathrm{~g} \cdot \mathrm{L}^{-1}$ DNTS, respectively. The switchgrass plants were grown in a growth chamber at $25{ }^{\circ} \mathrm{C}$ with a 16-hour light/8-hour dark photoperiod for 6 days, while $50 \mu \mathrm{L}$ of liquid medium were sampled each day. The contents of 2,4-DNT and DNTS were quantified by HPLC, as previously described (Xu et al., 2019).

\section{Transcriptome analysis of transgenic switchgrass plants}

The control and transgenic switchgrass plants at the E2 stage were treated for 2 days with $20 \mathrm{mg} \cdot \mathrm{L}^{-1} 2,4-$ DNT. Subsequently, the root tissues were collected for transcriptome analysis by RNA sequencing (RNA-seq). Transcriptome de novo assembly of switchgrass was performed directly on the set of sequenced reads using the Trinity 2.4.0 (Broad Institute, Boston, USA). Raw Illumina pair-end reads were trimmed using FastQC including the Q20, Q30, and GC-content of the clean data to obtain high quality reads. All assembled unigenes were aligned against the non-redundant (Nr) database, GO, SwissProt, KEGG, and eggnog databases using DIAMOND (Crystal Impact GbR, Bonn, Germany) with a threshold of E-value $<0.00001$. The differentially expressed unigenes were selected with $\log 2$ (fold change) [?]1 and with statistical significance ( $p$ [?] 0.05) by $\mathrm{R}$ package edgeR. Furthermore, GO and KEGG enrichment analysis were used for data mining.

\section{Statistical analysis}

Switchgrass plants were propagated by transferring the same number of tillers into each pot. Three copies of each line were grown in 1-gallon pots. In this study, the control switchgrass plants and two representative NfsI overexpressing transgenic lines were selected for further investigation of root length, ROS contents, 2,4DNT and DNTS uptake, as well as transcriptome profiling. Each sample included at least three vegetatively propagated copies. Data from each treatment were subjected to an analysis of variance (ANOVA). The significance of the treatments was tested at the $p$ [?]0.05 level. Standard errors were provided in all tables and figures as appropriate. All statistical analyses were performed using the SPSS package (SPSS Inc., 
Chicago, IL, USA).

\section{Results}

\section{Biodegradation metabolites of 2,4-DNT and DNTS transformed by NfsI in vitro}

We initially conducted codon optimization forEnterobater cloacae $N f s I$ to improve its expression in switchgrass (Fig. S1 ). Thereafter, we produced the recombinant NfsI in E.coli and examined the biodegradation metabolites of 2,4-DNT by LC-PDA/ESI-MS/MS. Our results showed that the NfsI transformed 2,4-DNT in 2/4-hydroxyl amino-4/2-mononitrotoluene (HAMNT, Peak 1) through a two-electron reduction (Fig. 1A, Fig. S2A, E ). Furthermore, HAMNT was reduced to 2/4-amino-4/2-mononitrotoluene (AMNT, Peak 2) or formed a novel compound by autonomous polymerization (Peak 3) (Fig. 1A, B, Fig. S2B, C, F, Fig. S3 ). In addition, we analyzed the biodegradation metabolites of DNTS and found that the NfsI only transformed DNTS into 4-hydroxyl amino-4/2-mononitrotoluene-3- $\mathrm{SO}_{3}{ }^{-}$(HAMNTS, Peak 4) (Fig. 1C, D, Fig. S2D, G ).

\section{NfsI had higher catalytic efficiency toward2,4-DNT than DNTS in vitro}

To evaluate the catalytic efficiency of NfsI toward 2,4-DNT and DNTS, we next examined NfsI enzyme kinetic characterization in vitro . Compared with DNTS, the NfsI had lower Kmtoward 2,4-DNT (256.9 +$68.9 \mu \mathrm{M}$ vs. $2814.1 \pm 586.9 \mu \mathrm{M})$, while it had higher $V_{\max }$ toward 2,4 -DNT $\left(183.0 \pm 18.3 \mu \mathrm{M} \cdot \mathrm{s}^{-1} \cdot \mathrm{mg}^{-1} \mathrm{vs}\right.$. $\left.19.4 \pm 1.6 \mu \mathrm{M} \cdot \mathrm{s}^{-1} \cdot \mathrm{mg}^{-1}\right)($ Table 1$)$. Moreover, the NfsI had higher $K_{\text {cat }} / K m$ toward 2,4-DNT (6.83 \pm 0.29$)$ than DNTS $(0.07 \pm 0.22)$, suggesting that the NfsI is highly efficient at detoxifying 2,4-DNT rather than DNTS in vitro (Table 1 ). Thus, we selected 2,4-DNT as the NfsI substrate for further studies.

\section{Effects of $\mathrm{pH}$ value and NADPH concentration on NfsI enzymatic activity}

Both $\mathrm{pH}$ value and NADPH concentration had significant effects on NfsI enzymatic activities in vitro . Therefore, we next decided to examine the optimum $\mathrm{pH}$ and NADPH concentration for NfsI toward 2,4DNT. Our results showed that NfsI enzymatic activities initially increased and then decreased from $\mathrm{pH} 4.0$ to 9.0. The optimum $\mathrm{pH}$ was around 6.0 at which the NfsI reached its highest activity (Fig. 2A ). We further studied the effect of NADPH concentration on NfsI enzymatic activity at $\mathrm{pH}$ 6.0. The NfsI activity was dramatically elevated as the NADPH concentration increased from $50 \mu \mathrm{M}$ to $200 \mu \mathrm{M}$ until a limiting rate was reached (Fig. 2B ).

\section{Generation and identification of $N f s I$ overexpressing transgenic switchgrass plants}

To improve uptake efficiency of 2,4-DNT and DNTS in plants, we overexpressed the codon optimized NfsI in switchgrass plants. All transgenic lines were produced from a single genotypic embryogenic switchgrass callus line through Agrobacterium -mediated transformation, which excluded the potential influence of the genetic background of switchgrass on 2,4-DNT and DNTS tolerance as well as biodegradation. Eighteen independent positive transgenic switchgrass lines were identified by genomic PCR with $N f s I$ specific primers. The control plants were generated with the pANIC6B empty vector which was used as the backbone for constructing $N f s I$ -overexpressing vector. There was no obvious morphological or developmental difference between transgenic and control switchgrass plants in our greenhouse condition (Fig. 3A ).

\section{Overexpression of $N f s I$ in switchgrass enhanced plant tolerance against 2,4-DNT}

We next decided to study if engineering $N f s I$ in switchgrass has potential for remediation of 2,4-DNT. Two transgenic lines NfsI_OE-02 and -14 with highest $N f s I$ transcript abundance were selected for further investigation (Fig. 3B ). Firstly, we regenerated numerous plantlets from wild type, control, and transgenic switchgrass plants by immature inflorescence-derived callus cultures. These uniform plants were employed for examining the tolerance to 2,4-DNT. Our results showed that 2,4-DNT had significant impacts on the growth and development of wild type switchgrass plants. They exhibited obvious etiolation and wilting symptoms after 14 days when incubated with more than $5 \mathrm{mg} \cdot \mathrm{L}^{-1}$ 2,4-DNT (Fig. S4A ). In addition, as 2,4-DNT concentration increased from 2 to $40 \mathrm{mg} \cdot \mathrm{L}^{-1}$, their root length decreased gradually, while the ROS content increased (Fig. S4B, C ). Furthermore, we measured the root length and ROS content of $N f s I$ overexpressing 
transgenic switchgrass plants. Compared with control plants, the root length of transgenic switchgrass plants increased by $32.2-46.1 \%$, and their ROS contents decreased by $21.8-35.6 \%$ at a high 2,4-DNT level $\left(20 \mathrm{mg} \cdot \mathrm{L}^{-1}\right)$ (Fig. 4 ). Therefore, our results indicate that the $N f s I$ overexpressing transgenic switchgrass plants have a higher capacity to tolerate 2,4-DNT than control plants.

\section{Overexpression of $N f s I$ in switchgrass improved the uptake rate of 2,4-DNT}

A liquid culture system was employed to examine uptake of 2,4-DNT by the $N f s I$ overexpressing transgenic switchgrass plants. We chose $20 \mathrm{mg} \cdot \mathrm{L}^{-1} 2,4$-DNT as the initial concentration based on the previous 2,4-DNT tolerance analysis in wild type switchgrass. The culture medium containing $20 \mathrm{mg} \cdot \mathrm{L}^{-1} 2,4$-DNT without switchgrass plants was used as a blank control. After 2 days, the control switchgrass plants removed $24.9 \%$ of 2,4-DNT from the culture medium, whereas NfsI_OE-02 and -14 transgenic lines removed $66.7 \%$ and $64.3 \%$ of 2,4-DNT from the culture medium, respectively (Fig. 5A ). After 6 days, the control switchgrass plants removed $56.3 \%$ of 2,4 -DNT from the culture medium (Fig. 5A ). However, they displayed serious symptoms of toxicity including growth stunting and leaf wilting, which prevented further removal of 2,4-DNT (Fig. S5A ). In contrast, $94.1 \%$ of 2,4-DNT was taken up by the transgenic switchgrass plants after 6 days (Fig. 5A ). Moreover, their growth was barely affected by 2,4-DNT treatment (Fig. S5B ). In addition, we compared the uptake rate of DNTS between control and transgenic switchgrass plants (Fig. 5B ). Our results showed that overexpressing $N f_{s} I$ in switchgrass did not improve the uptake rate of DNTS.

\section{Overexpression of $N f s I$ in switchgrass had global effects on expression profiling of genes re- sponsible for 2,4-DNT}

To assess the global effects of engineering $N f_{s} I$ in switchgrass on expression profiling of genes responsible for 2,4-DNT, we first examined the transcriptome of root samples of control switchgrass plants that were exposed for 2 days in a liquid culture medium supplied with $20 \mathrm{mg} \cdot \mathrm{L}^{-1}$ 2,4-DNT. The RNA-seq analysis revealed that 2,4-DNT treatment induced 4,740 out of $85,522(5.5 \%)$ genes differentially expressed in the control plants, with 3,002 genes upregulated and 1,738 genes downregulated, respectively (Fig. S6A, Table S2 ). The differentially expressed genes (DEGs) were subjected to Gene Ontology (GO) analysis, revealing that most enrichment pathways were related to the plasma membrane, oxidation-reduction process, toxin catabolic process, and secondary metabolites biosynthesis (Fig. S6B ).

We further compared the transcriptome profiling of treated and untreated control (CP-T and CP-UT) and transgenic (TP-T and TP-UT) switchgrass plants. The matrix analysis of the four sets of DEGs revealed that 732 genes clustered in intersection IV, which were upregulated in CP-T/CP-UT intersecting set but downregulated in TP-T/CP-T intersecting set (Fig. 6A ). Moreover, there were 353 genes in intersection VI, which were downregulated in CP-T/CP-UT intersecting set but upregulated in TP-T/CP-T intersecting set (Fig. 6A ). Our results suggest that overexpression of $N f s I$ in switchgrass can alleviate expression changes of $22.9 \%$ of the genes responsible for 2,4-DNT stress (Fig. 6A ). These genes were categorized into biological processes, and mainly enriched stress response, metabolic biosynthesis, gene expression regulation and protein modification, and growth-development response (Fig. 6B ). It is noteworthy that most of the genes among 353 candidates were involved in plant morphological formation and regulation processes (Fig. 6C and Table S3 ), implying that NfsI may promote recovery from the damage resulted from 2,4-DNT stress. In contrast, most of the 732 candidate genes participated in the secondary metabolism process of the plant. This finding suggests that some metabolic genes involved in 2,4-DNT detoxification were activated in switchgrass (Fig. 6D and Table S3 ).

\section{Discussion}

2,4-DNT is an important intermediate to produce polyurethane foams and dyes. It is also found in the environment as a mixture with TNT since 2,4-DNT is a byproduct of TNT biosynthesis. Although DNT and TNT are broadly used in civilian and military activities, both are highly toxic xenobiotics that threaten human health and environmental safety. Phytoremediation is a low-cost technique to clean up these nitroaromatic compounds (Doty, 2008). Previous studies have suggested that engineering bacterial NfsI in plants can detoxify TNT efficiently (Van Dillewijn et al., 2008; Zhang et al., 2017; Hannink et al., 2001). However, 
it remains unknown if this strategy could be employed for detoxification of DNT and its derivatives. It had been shown thatEnterobater cloacae NfsI, a member of the group B type I NRs, can convert both TNT and DNT in vitro. A recent study also indicates that the cleanup rate of $N$. tabacum plants for 2,4-DNT is significantly increased after inoculation with 2,4-DNT degrading P. putida strains (Akkaya, 2020). These works shed light on the effectiveness of genetically modifying plants for DNT remediation. Switchgrass is a perennial tall grass with great potential for phytoremediation of nitroaromatic compounds (Zhang et al., 2019). Unfortunately, overexpression of Enterobater cloacae NfsI in switchgrass plants did not improve their resistance to TNT. Thus, challenges remain for engineering $N f s I$ in switchgrass for the detoxification of DNT and its derivatives.

It has been suggested that the expression levels of genes driven by $35 \mathrm{~S}$ promoter in switchgrass are lower than maize ubiquitin (ZmUbq) promoter (Mann et al., 2011). Therefore, we re-overexpressed $N f s I$ in switchgrass under the control of ZmUbq promoter to increase $N f_{s} I$ transcript abundance. Moreover, we employed a codon optimization technique to improve transcription and translation efficiency of $N f s I$ in switchgrass since $N f_{s} I$ is a bacterial derived $N R$. Finally, we generated $N f s I$ overexpressing transgenic switchgrass plants and improved their tolerance and detoxification to 2,4-DNT. The uptake rate of 2,4-DNT increased from $\sim 56 \%$ in the control plants to $\sim 94 \%$ in transgenic lines NfsI_OE-02 and -14 (i.e., a relative increase of $67 \%$ ). Compared with 2,4-DNT, NfsI exhibited little conversion capacity to DNTS neither in vitro norin vivo, implying that sulfonate has a crucial effect on nitro reduction of 2,4-DNT. Therefore, the enzyme specificity to nitroaromatic compounds should be fully considered before engineering $N R s$ in plants for detoxification of these highly toxic pollutants.

NfsI reduces TNT to 2-hydroxyl-4,6-dinitrotoluene (HADNT) through two-electron reduction and then transforms HADNT to 2-amino-4,6-dinitrotoluene (ADNT). The reduction pathway of 2,4-DNT is similar to that of TNT, and both HAMNT and AMNT were produced during 2,4-DNT reduction catalyzed by NfsI. Most strikingly, a novel compound that likely formed by an autonomous polymerization was detected by LCPDA/ESI-MS/MS, suggesting HAMNT and its derivatives are the greatest sources of 2,4-DNT reduction. Compared with 2,4-DNT, DNTS were only transformed to HAMNTS by NfsI in vitro. Furthermore, the enzyme kinetics analysis indicates that NfsI has much higher affinity and catalytic efficiency for 2,4-DNT than DNTS. Given the fact that DNTS is a sulfonate of 2,4-DNT, we speculated that the presence of the sulfonate may block NfsI from binding to its substrate. Although the crystal structures of many bacterial NRs have been determined (Chauviac et al., 2012), the selectivity mechanism of NRs for their nitrated substrates warrants further investigation.

Overexpressing $N f_{S} I$ in switchgrass alleviated the impact of 2,4-DNT on root length and ROS production significantly. This is similar with the previous observation that overexpressing NfsI in Arabidopsis, tobacco, and poplar can partially restore the inhibition of TNT on root elongation and reduce ROS accumulation (Van Dillewijn et al., 2008; Hannink et al., 2001). Although the morphological and physiological responses of genetically modifying plants to nitroaromatic pollutants have been assessed widely, there are few studies involved in their transcriptome responses. One study indicated that TNT treatment can induce more than 500 genes that are differently expressed (at least 5-fold change) in wild type Arabidopsis roots. Serial analysis of gene expression further reveals multiphase mechanisms of TNT detoxification inArabidopsis including oxidative and reductive processes, conjugation reactions, and sequestering within the vacuole and/or cell wall (Reference, Ekman 2003). Genes involved in these detoxification processes were also differentially expressed in our control switchgrass treated with 2,4-DNT. It suggests that other plant species might employ a similar mechanism for detoxification of nitroaromatic pollutants. Furthermore, we found that overexpression of $N f s I$ in switchgrass was able to restore approximately $22.9 \%$ of DEGs induced by 2,4-DNT treatment by different extents. Among them, genes involved in reactions of oxidation and reduction, conjugation, and sequestering are worthy of functional characterization in the future.

In conclusion, the bacterial $N f s I$ was successfully overexpressed in switchgrass, which is a multiple purpose crop used for forage and biofuel production as well as phytoremediation. Overexpression of $N f_{s I}$ in switchgrass can remove $94.1 \%$ of 2,4 -DNT from liquid culture medium after 6 days which is 1.7 -fold higher than that 
of control plants. Moreover, our transcriptome analysis suggests that approximately $22.9 \%$ of switchgrass DEGs induced by 2,4-DNT might be involved in NfsI-mediated detoxification in switchgrass. Therefore, these genes are potential candidates for deciphering molecular mechanisms underlying switchgrass responses to nitroaromatic compounds. Further investigation will lead to more novel targets being discovered and engineered to improve switchgrass tolerance and detoxification to nitroaromatic pollutants in the future.

\section{Acknowledgements}

This work was supported by the Major Program of Shandong Province Natural Science Foundation (No. ZR2018ZB0213), Agricultural Variety Improvement Project of Shandong Province (No. 2019LZGC010), the Youth Innovation Promotion Association of CAS (2019213), QIBEBT and Dalian National Laboratory For Clean Energy (DNL),CAS (Grant: QIBEBT I201921 QIBEBT I201930).

\section{Author contributions}

CF and ZW designed the research; KS, YL, ZW, SJ and YW performed the experiments; CF, ZW, KS and YL analyzed the data; CF, ZW and KS wrote the manuscript.

\section{Conflict Interest}

The authors declare that they have no conflict of interest.

\section{References}

Akkaya, O., 2020. Nicotiana tabacum-associated bioengineered Pseudomonas putida can enhance rhizoremediation of soil containing 2,4-dinitrotoluene. 3 Biotech. 10, 1-11.

Alexandrova, K.S., Denchev, P.D., Conger, B. V., 1996. Micropropagation of switchgrass by node culture. Crop Sci. 36, 1709-1711.

Brentner, L.B., Mukherji, S.T., Walsh, S.A., Schnoor, J.L., 2010. Localization of hexahydro-1,3,5-trinitro1,3,5-triazine (RDX) and 2,4,6-trinitrotoluene (TNT) in poplar and switchgrass plants using phosphor imager autoradiography. Environ. Pollut. 158, 470-475.

Bryant, C., DeLuca, M., 1991. Purification and characterization of an oxygen-insensitive NAD(P)H nitroreductase from Enterobacter cloacae. J. Biol. Chem. 266, 4119-4125.

Bryant, C., Hubbard, L., McElroy, W.D., 1991. Cloning, nucleotide sequence, and expression of the nitroreductase gene fromEnterobacter cloacae. J. Biol. Chem. 266, 26-33.

Chauviac, F.X., Bommer, M., Yan, J., Parkin, G., Daviter, T., Lowden, P., Raven, E.L., Thalassinos, K., Keep, N.H., 2012. Crystal structure of reduced MsAcg, a putative nitroreductase from Mycobacterium smegmatis and a close homologue of Mycobacterium tuberculosisAcg. J. Biol. Chem. 287, 44372-44383.

Doty, S.L., 2008. Enhancing phytoremediation through the use of transgenics and endophytes. New Phytol. 179, 318-333.

Gandia-Herrero, F., Lorenz, A., Larson, T., Graham, I.A., Bowles, D.J., Rylott, E.L., Bruce, N.C., 2008. Detoxification of the explosive 2,4,6-trinitrotoluene in Arabidopsis: discovery of bifunctional $O$ - and $C$ glucosyltransferases. Plant J. 56, 963-974.

Gilbert, E.E., 1977. Recovery of organic values from TNT purification waste water. Propell. Explos. Pyrot. $2,118-125$.

Gong, P., Kuperman, R.G., Sunahara, G.I., 2003. Genotoxicity of 2,4- and 2,6-dinitrotoluene as measured by the Tradescantia micronucleus(Trad-MCN) bioassay. Mutat. Res-Gen. Tox. En. 538, 13-18.

Gunning, V., Tzafestas, K., Sparrow, H., Johnston, E.J., Brentnall, A.S., Potts, J.R., Rylott, E.L., Bruce, N.C., 2014. Arabidopsis glutathione transferases U24 and U25 exhibit a range of detoxification activities with the environmental pollutant and explosive 2,4,6-Trinitrotoluene. Plant Physiol. 165, 854-865. 
Hannink, N., Rosser, S.J., French, C.E., Basran, A., Murray, J.A.H., Nicklin, S., Bruce, N.C., 2001. Phytodetoxification of TNT by transgenic plants expressing a bacterial nitroreductase. Nat. Biotechnol. 19, $1168-1172$.

Hardin, C.F., Fu, C., Hisano, H., Xiao, X., Shen, H., Stewart, C.N., Parrott, W., Dixon, R.A., Wang, Z.Y., 2013. Standardization of switchgrass sample collection for cell wall and biomass trait analysis. Bioenergy. Res. 6, 755-762.

Johnson, G.R., Jain, R.K., Spain, J.C., 2002. Origins of the 2,4-dinitrotoluene pathway. J. Bacteriol. 184, 4219-4232.

Johnston, E.J., Rylott, E.L., Beynon, E., Lorenz, A., Chechik, V., Bruce, N.C., 2015. Monodehydroascorbate reductase mediates TNT toxicity in plants. Science. 349, 1072-1075.

Kim, H.Y., Song, H.G., 2005. Purification and characterization of NAD(P)H-dependent nitroreductase I from Klebsiella sp. C1 and enzymatic transformation of 2,4,6-trinitrotoluene. Appl. Microbiol. Biotechnol. 68, 766-773.

Kist, C.P., Scherer, C.E., Soares, M., Rodrigues, M.B., 2020. Biodegradation of nitroaromatic compounds in red water by white rot fungi pleurotus ostreatus and floridae. Rev. Ambient. Agua. 15, e2594.

Koder, R.L., Miller, A.F., 1998. Steady-state kinetic mechanism, stereospecificity, substrate and inhibitor specificity ofEnterobacter cloacae nitroreductase. Biochim. Biophys. Acta-Protein Struct. Mol. Enzymol. $1387,395-405$.

Lent, E.M., Crouse, L.C.B., Quinn, M.J., Wallace, S.M., 2012. Assessment of the in vivo genotoxicity of isomers of dinitrotoluene using the alkaline Comet and peripheral blood micronucleus assays. Mutat. Res-Gen. Tox. En. 742, 54-60.

Mann, D.G.J., King, Z.R., Liu, W., Joyce, B.L., Percifield, R.J., Hawkins, J.S., LaFayette, P.R., Artelt, B.J., Burris, J.N., Mazarei, M., Bennetzen, J.L., Parrott, W.A., Stewart, C.N., 2011. Switchgrass (Panicum virgatum L.) polyubiquitin gene (PvUbi1 and PvUbi2) promoters for use in plant transformation. BMC Biotechnol. 11.

Mann, D.G.J., LaFayette, P.R., Abercrombie, L.L., King, Z.R., Mazarei, M., Halter, M.C., Poovaiah, C.R., Baxter, H., Shen, H., Dixon, R.A., Parrott, W.A., Stewart, C.N., 2012. Gateway-compatible vectors for high-throughput gene functional analysis in switchgrass (Panicum virgatum L.) and other monocot species. Plant Biotechnol. J. 10, 226-236.

Rai, P.K., Kim, K.H., Lee, S.S., Lee, J.H., 2020. Molecular mechanisms in phytoremediation of environmental contaminants and prospects of engineered transgenic plants/microbes. Sci. Total Environ. 705.

Rao, M.R., Halfhill, M.D., Abercrombie, L.G., Ranjan, P., Abercrombie, J.M., Gouffon, J.S., Saxton, A.M., Stewart, C.N., 2009. Phytoremediation and phytosensing of chemical contaminants, RDX and TNT: identification of the required target genes. Funct. Integr. Genomics. 9, 537-547.

Rich, M.H., Sharrock, A. V, Hall, K.R., Ackerley, D.F., MacKichan, J.K., 2018. Evaluation of NfsA-like nitroreductases from Neisseria meningitidis and Bartonella henselae for enzyme-prodrug therapy, targeted cellular ablation, and dinitrotoluene bioremediation. Biotechnol. Lett. 40, 359-367.

Rickert, D.E., Butterworth, B.E., Popp, J.A., Krahn, D.F., 1984. Dinitrotoluene: Acute toxicity, oncogenicity, genotoxicity, and metabolism. Crit. Rev. Toxicol. 13, 217-234.

Roldan, M.D., Perez-Reinado, E., Castillo, F., Moreno-Vivian, C., 2008. Reduction of polynitroaromatic compounds: The bacterial nitroreductases. FEMS Microbiol. 32, 474-500.

Song, G., Yuan, S.X., Wen, X.H., Xie, Z.I., Lou, L.Q., Hu, B.Y., Cai, Q.S., Xu, B., 2018. Transcriptome analysis of Cd-treated switchgrass root revealed novel transcripts and the importance of HSF/HSP network in switchgrass Cd tolerance. Plant Cell Rep. 37, 1485-1497. 
Tognetti, V.B., Monti, M.R., Valle, E.M., Carrillo, N., Smania, A.M., 2007. Detoxification of 2,4dinitrotoluene by transgenic tobacco plants expressing a bacterial flavodoxin. Environ. Sci. Technol. 41, 4071-4076.

Tsai, T.S., 1991. Biotreatment of red water - a hazardous-waste stream from explosive manufacture - with fungal systems. Hazard. Waste Hazard. Mater. 8, 231-244.

Van Dillewijn, P., Couselo, J.L., Corredoira, E., Delgado, A., Wittich, R.M., Ballester, A., Ramos, J.L., 2008. Bioremediation of 2,4,6-trinitrotoluene by bacterial nitroreductase expressing transgenic aspen. Env. Sci Technol. 42, 7405-7410.

Williams, E.M., Sharrock, A. V, Rylott, E.L., Bruce, N.C., MacKichan, J.K., Ackerley, D.F., 2019. A cofactor consumption screen identifies promising NfsB family nitroreductases for dinitrotoluene remediation. Biotechnol. Lett. 41, 1155-1162.

Wise, A.A., Liu, Z., Binns, A.N., 2006. Three methods for the introduction of foreign DNA into Agrobacterium. Methods Mol. Biol. 343, 43-53.

Wu, Z.Y., Cao, Y.P., Yang, R.J., Qi, T.X., Hang, Y.Q., Lin, H., Zhou, G.K., Wang, Z.Y., Fu, C.X., 2016. Switchgrass SBP-boxtranscription factors PvSPL1 and 2 function redundantly to initiate side tillers and affect biomass yield of energy crop. Biotechnol. Biofuels. 9, 101.

Xiong, W.D., Wu, Z.Y., Liu, Y.C., Li, Y., Su, K.L., Bai, Z.T., Guo, S.Y., Hu, Z.B., Zhang, Z.M., Bao, Y., Sun, J., Yang, G.F., Fu, C.X., 2019. Mutation of 4-coumarate: coenzyme A ligase 1 gene affects lignin biosynthesis and increases the cell wall digestibility in maizebrown midrib5 mutants. Biotechnol. Biofuels. 12.

Xu, W.J., Zhao, Q.L., Li, Z.L., Lu, X.Y., Han, S.Q., Ye, Z.F., 2019. Biodegradation of dinitrotoluene sulfonates and other nitro-aromatic compounds by Pseudomonas sp. X5 isolated from TNT red water contaminated soil. J. Clean. Prod. 214, 782-790.

Zajc, B., 1999. Synthesis of (+-)-trans-7,8-dihydrodiol of 6-fluoro-benzo[a]pyrene via hydroxyl-directed regioselective functionalization of substituted pyrene. J. Org. Chem. 64, 1902-1907.

Zhang, L., Routsong, R., Nguyen, Q., Rylott, E.L., Bruce, N.C., Strand, S.E., 2017a. Expression in grasses of multiple transgenes for degradation of munitions compounds on live-fire training ranges. Plant Biotechnol. J. 15, 624-633.

Zhang, L., Rylott, E.L., Bruce, N.C., Strand, S.E., 2019. Genetic modification of western wheatgrass (Pascopyrum smithii) for the phytoremediation of RDX and TNT. Planta. 249, 1007-1015.

Zhang, L., Rylott, E.L., Bruce, N.C., Strand, S.E., 2017b. Phytodetoxification of TNT by transplastomic tobacco (Nicotiana tabacum ) expressing a bacterial nitroreductase. Plant Mol. Biol. 95, 99-109.

Ziganshin, A.M., Naumov, A. V., Suvorova, E.S., Naumenko, E.A., Naumova, R.P., 2007. Hydride-mediated reduction of 2,4,6-trinitrotoluene by yeasts as the way to its deep degradation. Microbiology. 76, 676-682.

Table 1. Enzyme kinetics analysis of recombinant NfsI against 2,4-DNT and DNTS in vitro.

\begin{tabular}{lllll}
\hline Substrate & $\mathrm{K}_{\mathrm{m}}(\mu \mathrm{M})$ & $\mathrm{V}_{\max }\left(\mu \mathrm{M} \cdot \mathrm{s}^{-1} \cdot \mathrm{mg}^{-1}\right)$ & $\mathrm{K}_{\text {cat }}\left(\mathrm{s}^{-1}\right)$ & $\begin{array}{l}\mathrm{K}_{\text {cat }} / \mathrm{K}_{\mathrm{m}} \\
\left(\mathrm{s}^{-1} \cdot \mathrm{mM}^{-1}\right)\end{array}$ \\
\hline 2,4 -DNT & $256.9 \pm 68.9$ & $183.0 \pm 18.3$ & $1.76 \pm 0.18$ & $6.83 \pm 0.29$ \\
DNTS & $2814.1 \pm 586.9$ & $19.4 \pm 1.6$ & $0.19 \pm 0.02$ & $0.07 \pm 0.22$ \\
\hline
\end{tabular}

Values are means \pm SE $(\mathrm{n}=3)$.

Figure Legends 
Fig. 1.M etabolites of 2,4-DNT and DNTS transformed by NfsI in vitro. HPLC analysis of metabolites of 2,4-DNT (A) and DNTS (B) transformed by the purified recombinant NfsI in vitro. The proposed pathway for the reduction of 2,4-DNT (C) and DNTS (D) by NfsI. Peak 1, 2/4-hydroxyl amino-4/2mononitrotoluene (HAMNT); Peak 2, 2/4-amino-4/2-mononitrotoluene (AMNT); Peak 3, a novel compound derived from HAMNT; Peak 4, 4-hydroxyl amino-4/2-mononitrotoluene-3-SO ${ }_{3}^{-}$(HAMNTS).

Fig. 2. Effects of pH value and NADPH concentration on NfsI enzymatic activity. The enzymatic activity of NfsI was examined at different $\mathrm{pH}$ values (A) and NADPH concentrations (B).

Fig. 3. Morphological and molecular characterization of transgenic switchgrass plants overexpressing bacterial $N f s I$. (A) Representative switchgrass plants from control (Ctrl) and transgenic (NfsI_OE-02 and -14) switchgrass plants are shown. (B) Quantitative real-time PCR analysis of NfsI transcript abundances in control and transgenic switchgrass plants. The control plants were generated with the pANIC6B empty vector. Stems at the R1 stage were collected. The expression level of switchgrassPvUbiq2 was used as the reference for normalization. Values are mean $\pm \mathrm{SE}(\mathrm{n}=3)$.

Fig. 4. Effect of 2,4-DNT on root length and ROS content of control and transgenic switchgrass plants. (A) Root length of the control and transgenic switchgrass plantlets were measured after 2,4-DNT treatment. (B) In situdetection of ROS in roots of control and transgenic switchgrass plantlets by DAB staining. (C) ROS content in roots of the control and transgenic switchgrass plantlets were measured after 2,4-DNT treatment. The control and transgenic (NfsI_OE-02 and -14) switchgrass plantlets were exposed to 0,2 , and $20 \mathrm{mg} \cdot \mathrm{L}^{-1} 2,4-\mathrm{DNT}$. After 14 days of treatment, the photographs of roots stained with NBT were taken. Root length as well as ROS content were also measured. Each sample included six vegetatively propagated copies. Values are mean $\pm \mathrm{SE}(\mathrm{n}=3)$.

Fig. 5. Uptake of 2,4-DNT and DNTS by control and transgenic switchgrass plants. (A) 2,4-DNT concentration in $1 / 2 \mathrm{MS}$ liquid culture medium with time. The control and transgenic switchgrass plants at E2 stage were exposed to 1/2 MS liquid medium supplied with $20 \mathrm{mg} \cdot \mathrm{L}^{-1} 2,4$-DNT, respectively. The blank was $1 / 2 \mathrm{MS}$ liquid medium supplied with $20 \mathrm{mg} \cdot \mathrm{L}^{-1} 2,4-\mathrm{DNT}$ without plants. (B) DNTS concentration in liquid culture medium with time. The control and transgenic switchgrass plants at E2 stage were exposed to 1/2 MS liquid medium supplied with $1000 \mathrm{mg} \cdot \mathrm{L}^{-1} \mathrm{DNTS}$, respectively. The blank was $1 / 2 \mathrm{MS}$ liquid medium supplied with $1000 \mathrm{mg} \cdot \mathrm{L}^{-1}$ DNTS without plants.

Fig. 6. Transcriptome analysis of transgenic switchgrass plants responses to 2,4-DNT treatment. (A) Venn diagram and intersecting sets analysis for upregulated and downregulated genes among the control and transgenic switchgrass plants with and without 2,4-DNT treatment. CP-UT, control plants without 2,4-DNT treatment; CP-T, control plants with $20 \mathrm{mg} \cdot \mathrm{L}^{-1} 2,4$-DNT treatment; TP-UT, transgenic plants (NfsI_OE-02 and -14) without 2,4-DNT treatment; TP-T, transgenic plants (NfsI_OE-02 and -14) with $20 \mathrm{mg} \cdot \mathrm{L}^{-1} 2,4$-DNT treatment for 2 days. The horizontal bars represent different intersections of four sets of samples (I-VIII). The vertical bars represent the gene number of the intersection. (B) Gene ontology term enrichment of differentially expressed genes (DEGs) of intersection IV and VI. (C) KEGG pathway enrichment of DEGs in intersection VI. (D) KEGG pathway enrichment of DEGs in intersection IV. The control and transgenic (NfsI_OE-02 and -14) plantlets were exposed to 0 and $20 \mathrm{mg} \cdot \mathrm{L}^{-1} 2,4$-DNT, respectively. After 2 days treatment, total RNAs were extracted and subjected to transcriptome analysis through RNA-sequencing. Each sample included three vegetatively propagated copies. Values are mean $\pm \mathrm{SE}(\mathrm{n}=$ $3)$.

\section{Supporting Information}

Table S1. Primers used in this study.

Table S2. Overview of differentially expressed genes between control and transgenic switchgrass plants.

+ CP-UT, control plants without 2,4-DNT treatment; CP-T, control plants with $20 \mathrm{mg}^{*} \mathrm{~L}^{-1} 2,4$-DNT treatment for 2 days; TP-UT, transgenic plants (NfsI_OE-02 and -14) without 2,4-DNT treatment; TP-T, trans- 
genic plants (NfsI_OE-02 and -14) with $20 \mathrm{mg}^{*} \mathrm{~L}^{-1}$ 2,4-DNT treatment for 2 days.

Table S3. Expression profiles of two intersections (IV and VI) involved in NfsI-mediated detoxification of 2,4-DNT in switchgrass.Fig. S1. Alignmentof nucleotide sequences of the wild

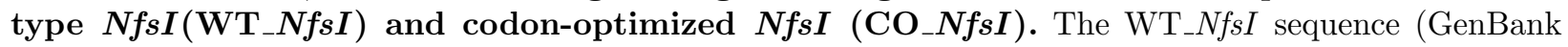
accession No. M6308.1) was retrieved from the Enterobacter cloacae genome sequence. The nucleotide sequence of $\mathrm{WT}_{-} N f_{s} I$ was optimized based on the codon preference of switchgrass and the CO_NfsI was synthesized.

Fig. S2. UV-Vis and mass spectra of intermediates of 2,4-DNT transformed by NfsI in vitro. (A-D) The UV-Vis spectra of peak 1-4. (E-G) The mass spectra of peak 1, 2, and 4.

Fig. S3. Autoformation of peak 3 from peak 1. (A) HPLC analysis of peak 3 autoformation with time. (B) The concentration of peak 1 and 3 with time. Peak 1 (HAMNT) was produced from the NfsI-mediated reduction reaction and purified by HPLC.

Fig. S4. Effect of 2,4-DNT on root length and ROS content of wild type switchgrass plants. (A) The wild type switchgrass plantlets were grown on MS0 medium supplied with various concentrations of 2,4-DNT for 14 days. (B) Root length of the wild type switchgrass plantlets were measured after 2,4-DNT treatment. (C) ROS content of the wild type switchgrass plantlets were measured after 2,4-DNT treatment.

Fig. S5. Impact of 2,4-DNT on root growth and development.Roots morphology of control (A) and transgenic (B) switchgrass plants after 6 days incubation with $20 \mathrm{mg}^{*} \mathrm{~L}^{-1}$ 2,4-DNT.

Fig. S6.Transcriptome analysis of control switchgrass plants responses to 2,4-DNT treatment. Analysis of volcano plots (A) and gene ontology term (B) of differentially expressed genes (DEGs) between control switchgrass plants with and without 2,4-DNT treatment. The control switchgrass plantlets were exposed to $20 \mathrm{mg}^{*} \mathrm{~L}^{-1}$ 2,4-DNT. After 2 days of treatment, total RNAs were extracted and subjected to transcriptome analysis through RNA-sequencing. Each sample included three vegetatively propagated copies. Values are mean $+-\mathrm{SE}(\mathrm{n}=3)$.
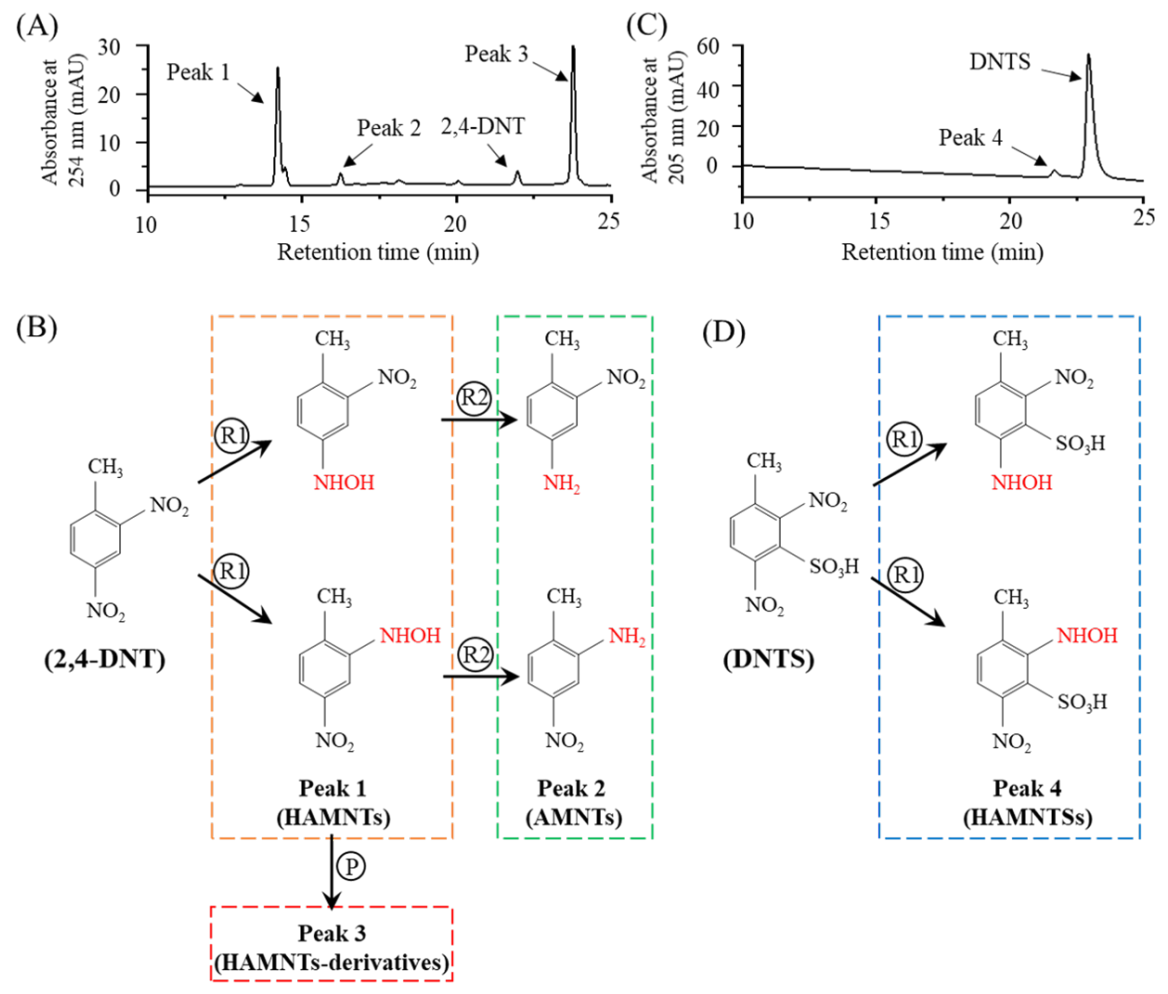
Fig. 1. Metabolites of 2,4-DNT and DNTS transformed by NfsI in vitro. HPLC analysis of metabolites of 2,4-DNT (A) and DNTS (B) transformed by the purified recombinant NfsI in vitro. The proposed pathway for the reduction of 2,4-DNT (C) and DNTS (D) by NfsI. Peak 1, 2/4-hydroxyl amino-4/2mononitrotoluene (HAMNT); Peak 2, 2/4-amino-4/2-mononitrotoluene (AMNT); Peak 3, a novel compound derived from HAMNT; Peak 4, 4-hydroxyl amino-4/2-mononitrotoluene-3-SO ${ }_{3}^{-}$(HAMNTS).
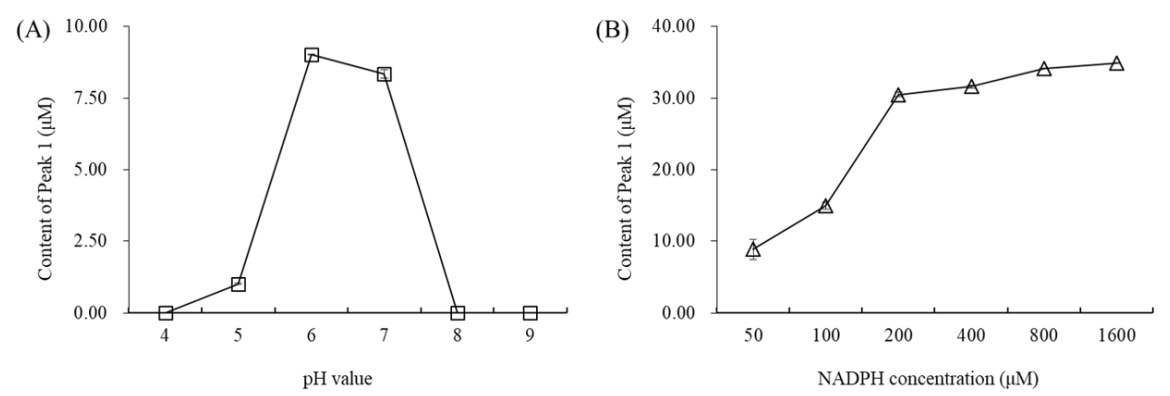

Fig. 2. Effects of $\mathbf{p H}$ value and NADPH concentration on NfsI enzymatic activity. The enzymatic activity of NfsI was examined at different $\mathrm{pH}$ values (A) and NADPH concentrations (B).

(A)

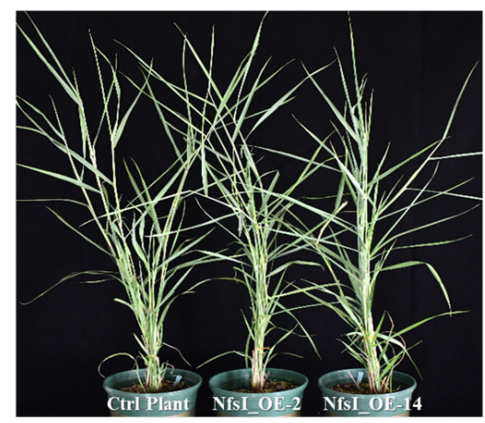

(B)

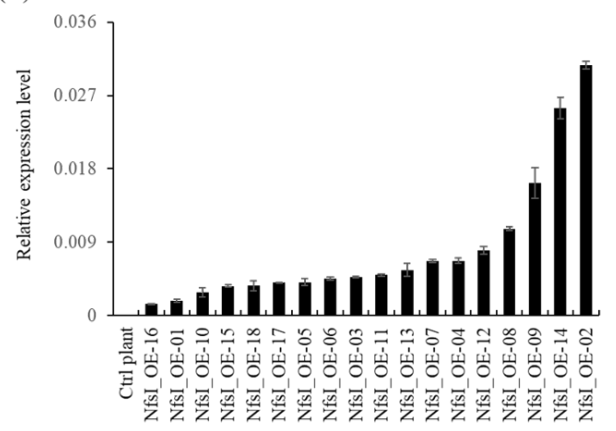

Fig. 3. Morphological and molecular characterization of transgenic switchgrass plants overexpressing bacterial NfsI. (A) Representative switchgrass plants from control (Ctrl) and transgenic (NfsI_OE-02 and -14) switchgrass plants are shown. (B) Quantitative real-time PCR analysis of NfsI transcript abundances in control and transgenic switchgrass plants. The control plants were generated with the pANIC6B empty vector. Stems at the R1 stage were collected. The expression level of switchgrass PvUbiq2 was used as the reference for normalization. Values are mean $\pm \mathrm{SE}(\mathrm{n}=3)$.
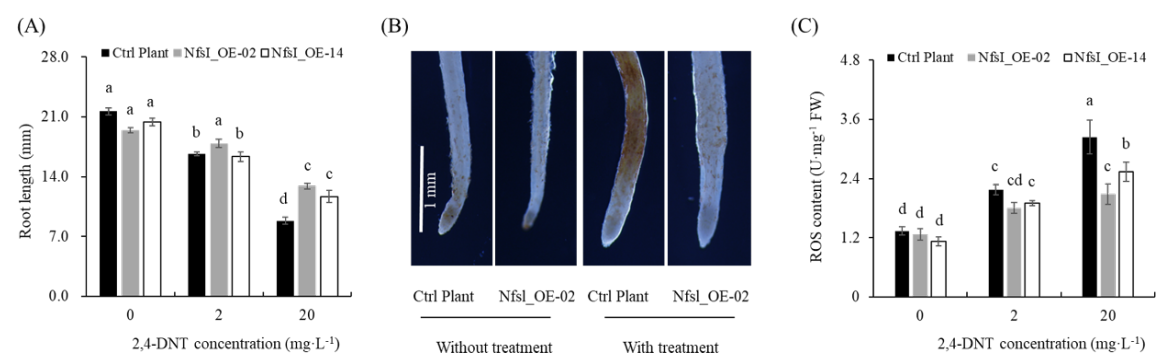

Fig. 4. Effect of 2,4-DNT on root length and ROS content of control and transgenic switchgrass plants. (A) Root length of the control and transgenic switchgrass plantlets were measured after 2,4-DNT treatment. (B) In situ detection of ROS in roots of control and transgenic switchgrass plantlets by DAB 
staining. (C) ROS content in roots of the control and transgenic switchgrass plantlets were measured after 2,4-DNT treatment. The control and transgenic (NfsI_OE-02 and -14) switchgrass plantlets were exposed to 0,2 , and $20 \mathrm{mg} \cdot \mathrm{L}^{-1} 2,4-\mathrm{DNT}$. After 14 days of treatment, the photographs of roots stained with NBT were taken. Root length as well as ROS content were also measured. Each sample included six vegetatively propagated copies. Values are mean $\pm \mathrm{SE}(\mathrm{n}=3)$.
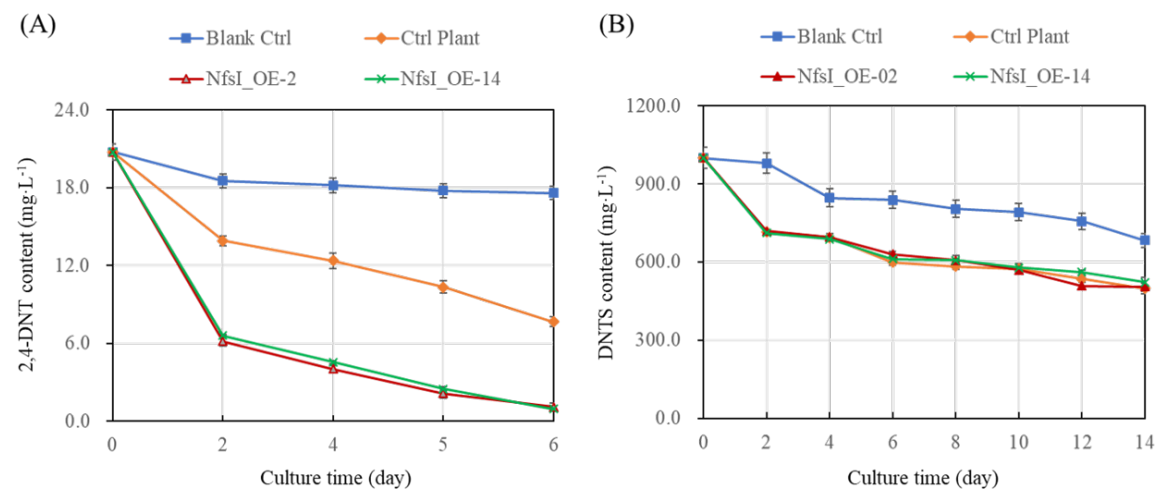

Fig. 5. Uptake of 2,4-DNT and DNTS by control and transgenic switchgrass plants. (A) 2,4 -DNT concentration in $1 / 2 \mathrm{MS}$ liquid culture medium with time. The control and transgenic switchgrass plants at E2 stage were exposed to 1/2 MS liquid medium supplied with $20 \mathrm{mg} \cdot \mathrm{L}^{-1} 2,4$-DNT, respectively. The blank was 1/2 MS liquid medium supplied with $20 \mathrm{mg} \cdot \mathrm{L}^{-1}$ 2,4-DNT without plants. (B) DNTS concentration in liquid culture medium with time. The control and transgenic switchgrass plants at E2 stage were exposed to $1 / 2 \mathrm{MS}$ liquid medium supplied with $1000 \mathrm{mg} \cdot \mathrm{L}^{-1}$ DNTS, respectively. The blank was $1 / 2 \mathrm{MS}$ liquid medium supplied with $1000 \mathrm{mg} \cdot \mathrm{L}^{-1}$ DNTS without plants
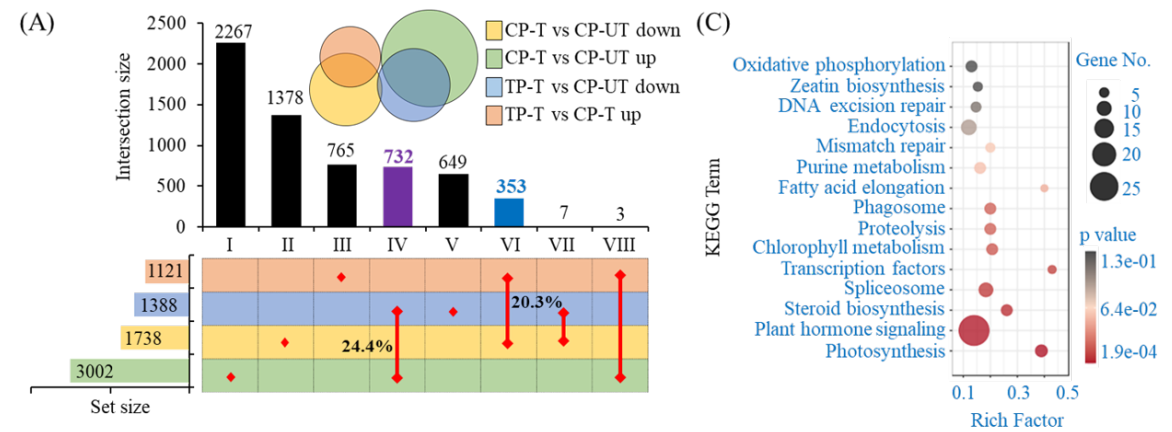

(B)

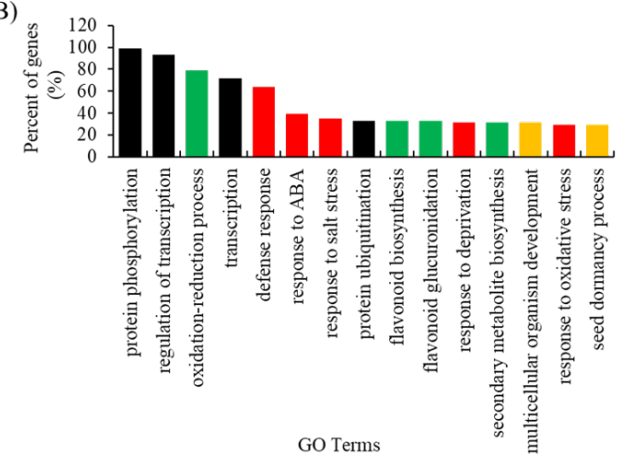

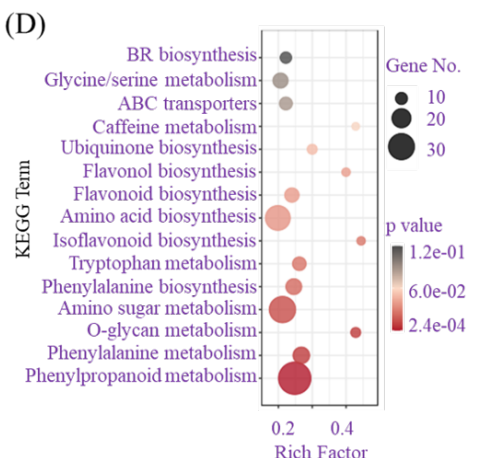

Fig. 6. Transcriptome analysis of transgenic switchgrass plants responses to 2,4-DNT treatment. (A) Venn diagram and intersecting sets analysis for upregulated and downregulated genes among 
the control and transgenic switchgrass plants with and without 2,4-DNT treatment. CP-UT, control plants without 2,4-DNT treatment; CP-T, control plants with $20 \mathrm{mg} \cdot \mathrm{L}^{-1}$ 2,4-DNT treatment; TP-UT, transgenic plants (NfsI_OE-02 and -14) without 2,4-DNT treatment; TP-T, transgenic plants (NfsI_OE-02 and -14) with $20 \mathrm{mg} \cdot \mathrm{L}^{-1} 2,4$-DNT treatment for 2 days. The horizontal bars represent different intersections of four sets of samples (I-VIII). The vertical bars represent the gene number of the intersection. (B) Gene ontology term enrichment of differentially expressed genes (DEGs) of intersection IV and VI. (C) KEGG pathway enrichment of DEGs in intersection VI. (D) KEGG pathway enrichment of DEGs in intersection IV. The control and transgenic (NfsI_OE-02 and -14) plantlets were exposed to 0 and $20 \mathrm{mg} \cdot \mathrm{L}^{-1} 2,4$-DNT, respectively. After 2 days treatment, total RNAs were extracted and subjected to transcriptome analysis through RNA-sequencing. Each sample included three vegetatively propagated copies. Values are mean $\pm \mathrm{SE}(\mathrm{n}=$ $3)$. 